Nervenarzt 2010 [Suppl 1] $\cdot 81: 41-41$

DOI 10.1007/s00115-010-3154-x

c) Springer-Verlag 2010
C. Koch · C.A.B. Eienbröker · L. Meyer · K. Hülsmeier · B. von Hagen · B. Tackenberg Klinische Neuroimmunologie, Klinik für Neurologie, Philipps-Universität-AG, Marburg

\title{
Krankheits- und Stressverarbeitung bei IFN- $\beta$-behandelten Patienten mit MS oder CIS
}

\section{Ziel des Forschungsprojektes}

Patienten, die an einer MS leiden, sind zu unterschiedlichen Zeitpunkten ihrer Krankengeschichte Stress ausgesetzt. Sowohl die Diagnosestellung als auch der Beginn einer Immuntherapie oder rezidivierende Krankheitsschübe können als Auslöser von Stress angesehen werden. Im Rahmen der hier vorgestellten prospektiven, kontrollierten Studie wurden Stresserleben und Copingstrategien nach Einleitung einer immunmodulatorischen Therapie mit IFN $\beta$-1b über 12 Monate seriell verglichen mit Daten von Patienten, die sich gegen eine Therapie entschieden hatten.

\section{Methode}

62 Patienten mit CIS verdächtig auf MS oder neu diagnostizierter RRMS wurden über einen Zeitraum von 18 Monaten rekrutiert. Alle Patienten hatten eine Indikation für den Beginn einer immunmodulatorischen Therapie mit IFN $\beta-1 b$. Nach standardisiertem ärztlichem Aufklärungsgespräch entschieden sich 41 Patienten für die Durchführung einer solchen, 21 Patienten entschieden sich dagegen und wurden in die unbehandelte Kontrollgruppe eingeschlossen.

Bei allen Patienten wurden in 3-monatlichem Abstand über 1 Jahr Verlaufskontrollen durchgeführt. Neben der Dokumentation des klinischen Verlaufs wurden Daten zur Depressivität/Fatigue, zu Copingstrategien, zum allgemeinen
Stressniveau und zur Lebensqualität erhoben.

Signifikanz wurde zweiseitig mittels Mann-Whitney-Test auf dem 5\%-Testniveau unter Verwendung von SPSS 17.0 getestet.

\section{Ergebnisse}

Die demografischen und klinischen Charakteristika beider Gruppen unterschieden sich nicht hinsichtlich Alter, Geschlecht und jährlicher Schubrate. Allerdings waren unbehandelte Patienten hinsichtlich des Ausgangs-EDSS stärker betroffen und hatten häufiger einen multifokalen Beginn der Symptomatik. In beiden Gruppen ließ sich zeigen, dass die Frage des Therapiebeginns als Stressor empfunden wurde $(\mathrm{p}<0,001)$. Unbehandelte Patienten zeigten ein geringeres $\mathrm{Ma}$ an Ängstlichkeit $(\mathrm{p}<0,005)$ und Depressivität $(\mathrm{p}<0,005)$ und verfolgten signifikant häufiger Bagatellisierung ( $\mathrm{p}<0,022)$ und Vermeidung ( $\mathrm{p}<0,0001)$ als Copingstrategien.

\section{Fazit}

Inwieweit eine therapeutische Modulation von Copingstrategien zum Zeitpunkt der Diagnosestellung die Entscheidung zum Beginn einer adhärenten immunmodulatorischen Therapie und den damit zu erwartenden günstigen Einfluss auf die Krankheitsentwicklung begünstigen würde, wird Gegenstand zukünftiger Interventionsstudien sein.

\section{Korrespondenzadresse \\ Dr. B. Tackenberg \\ Klinische Neuroimmunologie, Klinik für Neurologie, Philipps-Universität-AG Rudolf-Bultmann-Straße 8, 35033 Marburg tackenbb@staff.uni-marburg.de}

Interessenkonflikt. Der korrespondierende Autor gibt an, dass die Interessenkonflikte „Vortragshonorar", "Reisekostenerstattung " und "Forschungsunterstützung" durch die Bayer Vital GmbH/Bayer Schering Pharma AG bestehen.

Der korrespondierende Autor weist zusätzlich auf folgende Beziehungen hin: Dieselben Kooperationen bestehen mit Teva-Pharma, Biogen-Idec, Merck-Serono, Novartis. 